\title{
Bounds for the Zeros of a Polynomial with Restricted Coefficients
}

\author{
Abdul Aziz, Bashir Ahmad Zargar \\ Department of Mathematics, University of Kashmir, Srinagar, India \\ Email: aaulauzeem@rediffmail.com, bazargar@gmail.com
}

Received April 18, 2011; revised May 18, 2011; accepted May 26, 2011

\begin{abstract}
In this paper we shall obtain some interesting extensions and generalizations of a well-known theorem due to Enestrom and Kakeya according to which all the zeros of a polynomial $P(z)=a_{n} z^{n}+\cdots+a_{1} z+a_{0}$ satisfying the restriction $a_{n} \geq a_{n-1} \geq \cdots \geq a_{1} \geq a_{0}>0$ lie in the closed unit disk.
\end{abstract}

Keywords: Polynomial; Bounds; Zeros

\section{Introduction and Statement of Results}

The following results which is due to Enestrom and Kakeya [1] is well known in the theory of the location of the zeros of polynomials.

\section{THEOREM A. Let}

$$
P(z)=a_{n} z^{n}+a_{n-1} z^{n-1}+\cdots+a_{1} z+a_{0},
$$

be a polynomial of degree $\mathrm{n}$, such that

$$
a_{n} \geq a_{n-1} \geq \cdots \geq a_{1} \geq a_{0}>0,
$$

then $P(z)$ does not vanish in $|z|>1$.

In the literature [2-5] there exist some extensions and generalization of Enestrom-Kakeya Theorem. Joyal, Labelle and Rahman [6] extended this theorem to polynomials whose coefficients are monotonic but not necessarily non-negative by proving the following result:

THEOREM B. Let

$$
a_{n} \geq a_{n-1} \geq \ldots \geq a_{1} \geq a_{0}
$$

then the polynomial

$$
P(z)=a_{n} z^{n}+a_{n-1} z^{n-1}+\cdots+a_{1} z+a_{0}
$$

of degree $\mathrm{n}$ has all its zeros in

$$
|z| \leq \frac{1}{\left|a_{n}\right|}\left\{a_{n}-a_{0}+\left|a_{0}\right|\right\}
$$

Recently Aziz and Zarger [7] relaxed the hypothesis in several ways and among other things proved the following results:

THEOREM C. Let

$$
P(z)=a_{n} z^{n}+a_{n-1} z^{n-1}+\cdots+a_{1} z+a_{0}
$$

be the polynomial of degree $\mathrm{n}$, such that for some $k \geq 1$,

$$
k a_{n} \geq a_{n-1} \geq \cdots \geq a_{1} \geq a_{0}>0
$$

then $P(z)$ has all its zeros in

$$
|z+k-1| \leq k
$$

The aim of this paper is to prove some extensions of Enestrom-Kakeya Theorem (Theorem-A) by relaxing the hypothesis in various ways. Here we shall first prove the following generalization of Theorem $\mathrm{C}$ which is an interesting extension of Theorem A.

\section{Main Results}

THEOREM 1.1. Let

$$
P(z)=a_{n} z^{n}+a_{n-1} z^{n-1}+\cdots+a_{1} z+a_{0},
$$

be a polynomial of degree $\mathrm{n}$. If for some positive numbers $k$ and $\rho$ with $k \geq 1$, and $0<\rho \leq 1$

$$
k a_{n} \geq a_{n-1} \geq \cdots \geq \rho a_{0} \geq 0
$$

then all the zeros of $P(z)$ lie in the closed disk

$$
|z+k-1| \leq k+\frac{2 a_{0}}{a_{n}}(1-\rho)
$$

If we take $k=\frac{a_{n-1}}{a_{n}} \geq 1$, in Theorem 1.1 we obtain the following result which is a generalization of Corollary 2 ([7]).

COROLLARY 1. Let

$$
P(z)=a_{n} z^{n}+a_{n-1} z^{n-1}+\cdots+a_{1} z+a_{0},
$$

be a polynomials of degree $n$. If for some positive real 
number $\rho, 0<\rho \leq 1$

$$
a_{n} \leq a_{n-1} \geq a_{n-2} \geq \cdots \geq \rho a_{0}>0
$$

then all zeros of $P(z)$ lie in

$$
\left|z+\frac{a_{n-1}}{a_{n}}-1\right| \leq \frac{a_{n-1}}{a_{n}}+\frac{2 a_{0}}{a_{n}}(1-\rho)
$$

REMARK 1. Theorem 1.1 is applicable to situations when Enestrom-Kakeya Theorem gives no information. To see this consider the polynomial.

$$
P(z)=\alpha z^{n}+(\alpha-1) z^{n-1}+\cdots+(\alpha-1) z+\alpha,
$$

with $\alpha>1$ is a positive real number. Here EnestromKakeya Theorem is not applicable to $P(z)$ where as Theorem 1.1 is applicable with $k=1, \rho=\frac{n-1}{n}$ and according to our result, all the zeros of $P(z)$ lie in the disk.

$$
|z| \leq 1+\frac{1}{\alpha}, \alpha>1 .
$$

which is considerably better than the bound obtained by a classical result of Caushy ([4]) which states that all the zeros of $P(z)$ lie in

$$
|z| \leq 1+A
$$

where

$$
A=\max _{1 \leq j \leq n}\left|\frac{a_{n-j}}{a_{n}}\right|,
$$

Next, we present the following generalization of corollary 1 which includes Theorem 4 of [6] as a special case and considerably improves the bound obtained by Dewan and Bidkham ([8], Theorem1) for $t=0$ and $0 \leq k \leq n-1$.

THEOREM 1.2. Let

$$
P(z)=a_{n} z^{n}+a_{n-1} z^{n-1}+\cdots+a_{1} z+a_{0},
$$

be a polynomial of degree $\mathrm{n}$. If for some positive number $\rho, 0<\rho \leq 1$ and for some non-negative integer $\lambda, 0 \leq \lambda \leq n-1$

$$
a_{n} \leq a_{n-1} \leq \cdots \leq a_{\lambda+1} \leq a_{\lambda} \geq a_{\lambda-1} \geq \cdots \geq a_{1} \geq \rho a_{0},
$$

then all the zeros of $P(z)$ lie in

$$
\left|z+\frac{a_{n-1}}{a_{n}}-1\right| \leq \frac{1}{\left|a_{n}\right|}\left\{2 a_{\lambda}-a_{n-1}+(2-\rho)\left|a_{0}\right|-\rho a_{0}\right\}
$$

Applying Theorem 1.2 to $P(t z)$, we get the following result:

\section{COROLLARY 2. Let}

$$
P(z)=a_{n} z^{n}+a_{n-1} z^{n-1}+\cdots+a_{1} z+a_{0},
$$

be a polynomial of degree $n$. If for some positive numbers $t$ and $\rho$ with $0 .<\rho \leq 1$,

$$
t^{n} a_{n} \leq t^{n-1} a_{n-1} \leq \cdots \leq t^{\lambda} a_{\lambda} \geq \cdots \geq t a_{1} \geq t \rho a_{0}
$$

where $\lambda, 0 \leq \lambda \leq n-1$ is a non negative integer then all the zeros of $P(z)$, lie in

$$
\left|z+\frac{a_{n-1}}{a_{n}}-t\right| \leq \frac{t}{\left|a_{n}\right|}\left\{\left(\frac{2 a_{\lambda}}{t^{n-\lambda}}-\frac{a_{n-1}}{t}\right)+\frac{1}{t^{n}}\left((2-\rho)\left|a_{0}\right|+a_{0}\right)\right\}
$$

If we assume $a_{0}>0$, in Theorem 1.2, we obtain.

COROLLARY 3. Let

$$
P(z)=a_{n} z^{n}+a_{n-1} z^{n-1}+\cdots+a_{1} z+a_{0},
$$

be a polynomial of degree $n$. If for some positive numbers $\rho, 0<\rho<1$ and for same non-negative integer $\lambda$, $0 \leq \lambda \leq n-1$

$$
a_{n} \leq a_{n-1} \leq \cdots \leq a_{\lambda} \geq \cdots \geq a_{1} \geq \rho a_{0}>0
$$

then all the zeros of $P(z)$ lie in,

$$
\left|z+\frac{a_{n-1}}{a_{n}}-1\right| \leq \frac{1}{a_{n}}\left(2 a_{\lambda}-a_{n-1}+2(1-\rho) a_{0}\right)
$$

Finally we present all following generalization of Theorem B due to Joyal, Labelle and Rahman which includes Theorem A as a special case.

THEOREM 1.3. Let

$$
P(z)=a_{n} z^{n}+a_{n-1} z^{n-1}+\cdots+a_{1} z+a_{0},
$$

be a polynomial of degree $n$, It for some positive number $\rho, 0<\rho \leq 1$ and for some non-negative integer $\lambda, 0 \leq \lambda \leq n-1$

$$
a_{n} \leq a_{n-1} \leq \cdots \leq a_{\lambda} \geq \cdots \geq a_{1} \geq \rho a_{0}
$$

then all the zeros of $P(z)$ lie in

$$
|z| \leq \frac{2 a_{\lambda}-a_{n}+(2-\rho)\left|a_{0}\right|+\rho a_{0}}{\left|a_{n}\right|}
$$

REMARK 2. For $\rho=1$, Theorem 1.3 reduces to Theorem B.

\section{Proofs of the Theorems}

PROOF OF THEOREM 1.1. Consider

$$
\begin{aligned}
F(z) & =(1-z) P(z)=-a_{n} z^{n+1}+\left(a_{n}-a_{n-1}\right) z^{n}+\cdots+\left(a_{1}-a_{0}\right) z+a_{0}, \\
& =-a_{n} z^{n+1}+a_{n} z^{n}-k a_{n} z^{n}+\left(k a_{n}-a_{n-1}\right) z^{n}+\cdots+\left(a_{1}-\rho a_{0}\right) z+(\rho-1) a_{0} z+a_{0}
\end{aligned}
$$


then for $|z|>1$, we have

$$
\begin{aligned}
\begin{aligned}
|F(z)| & =\left|-a_{n} z^{n+1}+a_{n} z^{n}-k a_{n} z^{n}+\left(k a_{n}-a_{n-1}\right) z^{n}+\cdots+\left(a_{1}-\rho a_{0}\right) z+(\rho-1) a_{0} z+a_{0}\right| \\
& \geq\left|a_{n}\right||z|^{n}\left[|z+k-1|-\frac{1}{\left|a_{n}\right|}\left\{\left|\left(k a_{n}-a_{n-1}\right)+\left(a_{n-1}-a_{n-2}\right) \frac{1}{z}+\cdots+\left(a_{0}-p a_{0}\right) z(\rho-1) \frac{a_{0}}{z^{n}}+\frac{a_{0}}{z^{n}}\right|\right\}\right] \\
& \geq\left|a_{n}\right||z|^{n}\left[|z+k-1|-\frac{1}{\left|a_{n}\right|}\left\{k a_{n}-\rho a_{0}+(1-\rho)\left|a_{0}\right|+\left|a_{0}\right|\right\}\right] \\
& =\left|a_{n}\right||z|^{n}\left[|z+k-1|-\frac{1}{a_{n}}\left\{k a_{n}-\rho a_{0}+a_{0}+(1-\rho) a_{0}\right\}\right]>0,
\end{aligned} \\
\text { if }|z+k-1|>\frac{k a_{n}+2(1-\rho) a_{0}}{a_{n}}
\end{aligned}
$$

this shows that if $|z|>1$ then $|F(z)|>0$, if

$$
|z+k-1|>k+2(1-\rho) \frac{a_{0}}{a_{n}}
$$

therefore all the zeros of $F(z)$, whose modulus is greater than 1 lie in the closed disk

$$
\begin{aligned}
&|z+k-1| \leq k+2(1-\rho) \frac{a_{0}}{a_{n}} \begin{aligned}
\text { Pem 1.1. } \\
\text { PROOF OF THEOREM 1.2. Consider }
\end{aligned} \\
& \begin{aligned}
F(z) & =(1-z) P(z) \\
& =(1-z)\left(a_{n} z^{n}+a_{n-1} z^{n-1}+\cdots+a_{\lambda} z^{\lambda}+\cdots+a_{1} z+a_{0}\right), \\
& =-a_{n} z^{n+1}+\left(a_{n-\lambda} a_{n-1}\right) z^{n}+\cdots+\left(a_{\lambda-1}-a_{\lambda}\right) z^{\lambda+1}+\left(a_{\lambda}-z_{\lambda-1}\right) z^{\lambda}+\cdots+a_{1} z-a_{0} z+a_{0}
\end{aligned}
\end{aligned}
$$

therefore, for $|z|>1, \leq \lambda \leq n-1$, and $0<\rho<1$, we have

$$
\begin{aligned}
&|F(z)| \geq\left|a_{n} z^{n+1}-a_{n} z^{n}+a_{n-1} z^{n}\right|-\mid\left(a_{n-1}-a_{n-2}\right) z^{n-1}+\cdots+\left(a_{\lambda+1}-a_{\lambda}\right) z^{\lambda+1}+\left(a_{\lambda}-a_{\lambda-1}\right) z^{\lambda} \\
&+\cdots+\left(a_{1}-\rho a_{0}\right) z+\left(\rho a_{0}-a_{0}\right) z+a_{0} \mid \\
& \geq\left|a_{n}\right||z|^{n}\left|z+\frac{a_{n-1}}{a_{n}}-1\right|-|z|^{n}\left\{\left|a_{n-1}-a_{n-2}\right| \frac{1}{|z|}+\cdots+\left|a_{\lambda-1}-a_{\lambda}\right| \frac{1}{|z|^{n-\lambda-1}}+\left|a_{\lambda-1}-a_{\lambda}\right| \frac{1}{|z|^{n-\lambda}}\right. \\
&+\cdots+\left|a_{1}-\rho a_{0}\right| \frac{1}{|z|^{n-1}}+|1-\rho|\left|a_{0}\right| \frac{1}{|z|^{n-1}}+\frac{\left|a_{0}\right|}{\left.|z|^{n}\right\}} \\
&>\left|a_{n}\right||z|^{n}\left[\left|z+\frac{a_{n-1}}{a_{n}}-1\right|-\frac{1}{\left|a_{n}\right|}\left\{\left(a_{n-2}-a_{n-1}\right)+\left(a_{n-3}-a_{n-2}\right)+\left(a_{\lambda}-a_{\lambda+1}\right)\right.\right. \\
&\left.\left.+\left(a_{\lambda}-a_{\lambda-1}\right)+\cdots+\left(a_{1}-\rho a_{0}\right)+(1-\rho)\left|a_{0}\right|+\left|a_{0}\right|\right\}\right] \\
&=\left|a_{n}\right||z|^{n}\left[\left|z+\frac{a_{n-1}}{a_{n}}-1\right|-\frac{1}{\left|a_{n}\right|}\left\{2 a_{\lambda}-a_{n-1}+(2-\rho)\left|a_{0}\right|-\rho a_{0}\right\}\right]>0, \\
& \text { if }\left|z+\frac{a_{n-1}}{a_{n}}-1\right|>\frac{2 a_{\lambda}-a_{n-1}+(2-\rho)\left|a_{0}\right|-\rho a_{0}}{a_{n}}
\end{aligned}
$$

Therefore all the zeros of $F(z)$ whose modulus is greater than 1 lie in the circle. 


$$
\left|z+\frac{a_{n-1}}{a_{n}}-1\right| \leq \frac{2 a_{\lambda}-a_{n-1}+(2-\rho)\left|a_{0}\right|-\rho a_{0}}{a_{n}}
$$

But those zeros of $F(z)$ whose modulus is less than or equal to 1 already satisfy the Inequality (9).
Since all the zeros of $P(z)$ are also the zeros of $F(z)$, therefore it follows that all the zeros of $P(z)$ lie in the circle defined by (9). This completes the proof of Theorem 1.2 .

PROOF OF THEOREM 1.3. Consider

$$
\begin{aligned}
F(z) & =(1-z) P(z) \\
& =(1-z)\left(a_{n} z^{n}+a_{n-1} z^{n-1}+\cdots+a_{\lambda} z^{\lambda}+\ldots+a_{1} z+a_{0}\right) \\
& =-a_{n} z^{n+1}+\left(a_{n}-a_{n-1}\right) z^{n}+\cdots+\left(a_{\lambda+1} a_{\lambda}\right) z^{\lambda+1}+\left(a_{\lambda}-a_{\lambda-1}\right) z^{\lambda}+\cdots+a_{1} z-a_{0} z+a_{0}
\end{aligned}
$$

therefore, for $|z|>1,0 \leq \lambda \leq n-1$ and $0<\rho \leq 1$, we have

$$
|F(z)| \geq\left|a_{n} z^{n+1}\right|-\left|\left(a_{n}-a_{n-1}\right) z^{n}+\cdots+\left(a_{\lambda}-a_{\lambda-1}\right) z^{\lambda}+a_{1} z-a_{0} z+a_{0}\right|
$$

Proceeding similarly as in the proof of Theorem 1.2, we have

$$
\begin{aligned}
& |F(z)|>\frac{\left|a_{n}\right||z|^{n}\left\{|z|-2 a_{\lambda}-a_{n}+(2-\rho)\left|a_{0}\right|-\rho a_{0}\right\}}{a_{n}}>0, \\
& \text { if }|z|>\frac{2 a_{\lambda}-a_{n}+(2-\rho)\left|a_{0}\right|+\rho a_{0}}{\left|a_{n}\right|}
\end{aligned}
$$

therefore all the zeros of $F(z)$ whose modules is greater than 1 lie in the circle

$$
|z| \leq \frac{\left(2 a_{\lambda}-a_{n}\right)+(2-\rho)\left|a_{0}\right|+\rho a_{0}}{a_{n}}
$$

But those zeros of $F(z)$ whose modulus is $\leq 1$ already satisfy the (11). Since all the zeros of $P(z)$ are also the zero of $F(z)$, therefore it follows that all the zeros of $P(z)$ lie in circle defined by (11) and hence Theorem 1.3 is proved completed.

\section{Acknowledgements}

The authors are grateful to the refree for useful suggestions.

\section{REFERENCES}

[1] P. V. Krishnalah, “On Kakeya Theorem," Journal of Lon- don Mathematical Society, Vol. 20, No. 3, 1955, pp. 314319. doi:10.1112/jlms/s1-30.3.314

[2] A. Aziz and Q. G. Mohammad, "On the Zeros of a Certain Class of Polynomials and Related Analytic Functions," Journal of Mathematical Analysis and Applications, Vol. 75, No. 2, 1980, pp. 495-502. doi:10.1016/0022-247X(80)90097-9

[3] N. K. Govil and Q. I. Rahman, "On the Enestrom-Kakeya Theorem II," Tohoku Mathematical Journal, Vol. 20, No. 2, 1968, pp. 126-136. doi:10.2748/tmj/1178243172

[4] M. Marden, "Geometry of Polynomials," 2nd Edition, Vol 3, American Mathematical Society, Providence, 1966.

[5] G. V. Milovanovic, D. S. Mitrovic and Th. M. Rassias, "Topics in Polynomials, Extremal Problems Inequalities, Zeros," World Scientific, Singapore, 1994.

[6] A. Joyal, G. Labelle and Q. I. Rahman, "On the Location of Zeros of Polynomial," Canadian Mathematical Bulletin, Vol. 10, 1967, pp. 53-63. doi:10.4153/CMB-1967-006-3

[7] A. Aziz and B. A. Zargar, "Some Extensions of Enestrom Kakeya Theorem," Glasnick Matematicki, Vol. 31, 1996, pp. 239-244.

[8] K. K. Dewan and M. Bidkham, "On the Enestrom Kakeya Theorem," Journal of Mathematical Analysis and Applications, Vol. 180, No. 1, 1993, pp. 29-36. doi:10.1006/jmaa.1993.1379 